\title{
Le recueil au Moyen Âge. Le Moyen Âge central, sous la direction de Yasmina Foehr-Janssens et Olivier Collet
}

\section{Barbara Ferrari}

\section{CpenEdition}

\section{Journals}

Edizione digitale

URL: https://journals.openedition.org/studifrancesi/4553

DOI: $10.4000 /$ studifrancesi. 4553

ISSN: 2427-5856

\section{Editore}

Rosenberg \& Sellier

\section{Edizione cartacea}

Data di pubblicazione: 1 avril 2012

Paginazione: 113-114

ISSN: 0039-2944

\section{Notizia bibliografica digitale}

Barbara Ferrari, «Le recueil au Moyen Âge. Le Moyen Âge central, sous la direction de Yasmina FoehrJanssens et Olivier Collet», Studi Francesi [Online], 166 (I | LVI) | 2012, online dal 30 novembre 2015, consultato il 18 novembre 2021. URL: http://journals.openedition.org/studifrancesi/4553 ; DOI: https:// doi.org/10.4000/studifrancesi.4553

Questo documento è stato generato automaticamente il 18 novembre 2021.

\section{cc)}

Studi Francesi è distribuita con Licenza Creative Commons Attribuzione - Non commerciale - Non opere derivate 4.0 Internazionale. 


\title{
Le recueil au Moyen Âge. Le Moyen Âge central, sous la direction de Yasmina Foehr-Janssens et Olivier Collet
}

\author{
Barbara Ferrari
}

\section{NOTIZIA}

Le recueil au Moyen Âge. Le Moyen Âge central, sous la direction de Yasmina FOEHR-JANSSENS et Olivier ColLET, Turnhout, Brepols, 2010 («Texte, Codex \& Contexte», 8), pp. 304.

1 Jacqueline CERQUIGLINI-TOULET, nelle pagine che introducono i saggi qui presentati (Jeux du hasard et de l'intention: le recueil au Moyen Âge, pp. 7-9), sottolinea l'interesse crescente manifestato dalla critica negli ultimi dieci anni per le raccolte manoscritte medievali, interesse testimoniato da convegni e numeri monografici di riviste dedicati all'argomento. Anche questo volume, l'ottavo della collana «Texte, Codex \& Contexte», è il frutto di un colloquio su questo tema tenutosi a Ginevra nel 2007; al centro della riflessione i 'recueils organiques' - quelli cioè «qui ont bien existé comme tels au moyen âge» (p. 7) - prodotti tra i secoli XII e XIV; le raccolte della fine del medioevo sono invece state oggetto di studio in un altro convegno, a questo correlato, svoltosi a Louvain-laNeuve nello stesso anno, i cui atti sono riuniti nel n. 9 della medesima collezione (cfr. la rec. di Antonella Amatuzzi nel n. 163 di «Studi Francesi», pp. 150-152).

Il volume è diviso in due sezioni tematiche: la prima, dedicata a «Littérature, recueils narratifs et didactiques» (pp.11-156), comprende sette contributi e si apre con la presentazione di Wagih AZZAM, Olivier COLLET et Yasmina FOEHR-JANSSENS, dell'Università di Ginevra, che tracciano un bilancio critico dei risultati conseguiti dalla ricerca sui raggruppamenti testuali nel medioevo condotta nell'ambito del progetto Hypercodex (Mise en recueil et fonctionnalités de l'écrit, pp. 11-34). Nei saggi che seguono si alternano indagini su raccolte 'tematiche' e analisi dei contesti manoscritti di singole opere che gettano nuova luce sulla loro ricezione e consentono di affinarne l'interpretazione. 
3 Richard TRACHSLER (Observations sur les «recueils de fabliaux», pp. 35-46) propone di considerare il corpus dei manoscritti che trasmettono dei fabliaux non come un insieme monolitico ma come «un agglomérat de différents cas de figure» (p. 39) che richiedono l'applicazione di diverse chiavi interpretative. L'approccio quantitativo si dimostra particolarmente fecondo nell'individuare delle costanti che permettano di render conto dell'organizzazione delle raccolte.

4 Keith BUBSy (Le contexte manuscrit du "Songe d'Enfer" de Raoul de Houdenc, pp. 47-61) analizza la tradizione manoscritta del Songe d'Enfer (dieci testimoni) secondo la prospettiva delineata nella sua importante monografia Codex and Context: Reading Old French Verse Narrative in Manuscript (Amsterdam 2002). A suo avviso la diversità dei contesti manoscritti pone l'opera di Raoul de Houdenc al centro «d'un réseau intertextuel avec comme préoccupation principale l'inquiétude [...] sur le métier de poète comme métaphore de la vie humaine» (p. 60).

5 La particolarità del codice miscellaneo studiato da Milena MiKHAїLOVA (Le 'bien dire' $d u$ manuscrit BnF fr. 24301. Recueils et fleurs de rhétorique, pp. 63-90) consiste nel fatto che in esso si manifestano due diverse pratiche editoriali: quella della raccolta 'collettiva', che interessa l'intero manoscritto, e quella della raccolta 'personale', che concerne unicamente l'ultima sezione. In essa sono riunite le opere di Robert de Blois inserite nel Roman de Beaudous, probabilmente dallo stesso autore, secondo la tecnica dell'«enchassement». L'interpretazione dei testi e l'analisi della loro presentazione materiale convincono la studiosa che «la notion du discours est au centre de la composition des deux recueils» (p. 69).

6 Analizzando la «mise en recueil» di quattro romanzi 'atipici', trasmessi accanto ad opere a dominante didattica (Mise en recueil et typologie des genres aux XIII' et XIV siècles: romans atypiques et recueils polygénériques ("Biausdous", "Cristal et Clarie", "Durmart le Gallois" et "Mériadeuc"), pp. 91-111), Francis GINGRAS riflette sul loro statuto generico e mostra come essi si inseriscano a pieno titolo nel discorso 'anti-romanzesco' che si sviluppa nella seconda metà del XIII secolo.

7 Christopher LUCKEN (Les manuscrits du "Bestiaire d'Amours" de Richard de Fournival, pp.113-138) esamina la ricezione manoscritta del Bestiaire d'Amours. Nella maggior parte dei casi l'organizzazione delle raccolte in cui è inserito sfugge a ogni tentativo di interpretazione, ma in alcuni codici il contenuto e la disposizione dei testi «traduisent une authentique volonté d'organiser la tradition littéraire qu'ils sont chargés de transmettre» (p.138). La varietà dei criteri in base ai quali sono state costituite le diverse raccolte testimonia delle differenti letture di cui è stato oggetto il trattato di Richard de Fournival.

8 Chiude la sezione l'articolo di Amy Suzanne HENEVELD ("Chi commence d'amours», ou commencer pour finir: la place des arts d'aimer dans les manuscrits-recueils du XIII siècle, pp. 139-156), che, esaminando alcuni codici miscellanei del xIII secolo contenenti brevi testi sull'amore, ipotizza come chiave di lettura per alcune di queste raccolte quella di una didattica amorosa. Il fatto che questi 'arts d'aimer', spesso raggruppati alla fine dei manoscritti che li trasmettono, esercitino in qualche modo una funzione di 'chiusura', favorirebbe inoltre "une réflexion sur le role ambigu, et liminaire, de l'expérience amoureuse» (p. 155). 
9 Nella seconda sezione, «Sommes et manuscrits religieux» (pp. 157-268), che comprende solo cinque contributi, vengono prese in considerazione raccolte più omogenee che riuniscono testi religiosi o scientifici.

Brigitte Roux (L'encyclopédiste à l'œuvre: images de la compilation, pp. 157-181) riflette sulla figura dell'autore/compilatore delle grandi summe del xil secolo e sulle tecniche e gli strumenti impiegati nella pratica della compilazione attraverso l'analisi delle rappresentazioni dell'enciclopedista all'opera in una serie di codici miniati.

11 La tradizione manoscritta della prima redazione dell'Image du monde di Gossuin de Metz (1246) è indagata da Chantal CONNOCHIE-BOURGNE (Au temps des sommes, quelques recueils de textes didactiques, pp. 183-197), che si sofferma in particolare sulle raccolte della seconda metà del XIII secolo. La coerenza di questi recueils è assicurata dal carattere edificante dei testi che li compongono, la cui successione sembra studiata per formare un tutto le cui parti si completano o si amplificano a vicenda; solo la lettura di tutti i testi nell'ordine stabilito da chi ha organizzato la raccolta permetterà di comprendere appieno il senso dell'insieme.

Marie-Laure SAVOYE (Semis, transplantation et greffe: les techniques de la compilation dans le Rosarius, pp. 199-221) analizza in modo approfondito la composizione del ms. BnF, fr. 12483, meglio conosciuto come Rosarius. Sono via via presi in esame la struttura, il contenuto, le fonti e le tecniche di compilazione di questa grande raccolta dedicata alla Vergine che intreccia diverse tradizioni letterarie (le proprietates rerum, le raccolte di miracoli, i canzonieri).

13 Nei leggendari metodici francesi dei secoli XIII-XIV si incontrano spesso, in apertura, alcune leggende legate alla vita di Cristo. Anne-Françoise LABIE-LEURQUIN (Les hésitations du cycle christique du légendier $\mathrm{G}$ de Paul Meyer, pp. 223-235) si sofferma sul ciclo che figura in tutti i testimoni del cosiddetto leggendario $G$, studiandone la formazione, le possibili fonti e la mise en page. Viene messo in luce anche il metodo di lavoro del compilatore che, avendo a disposizione sia un leggendario metodico francese inaugurato da un ciclo cristico, sia una traduzione della Legenda aurea, non opta per il primo, nonostante l'analoga organizzazione, ma attinge ampiamente alla seconda, scegliendo uno a uno i testi da inserire.

14 Tra le opere di Gautier de Coinci, le preghiere hanno conosciuto una diffusione estremamente variegata. Alison STONES (Les prières de Gautier de Coinci, leur distribution et leur réception d'après la tradition manuscrite, pp.237-268) si interroga sulla loro distribuzione cronologica e geografica e sulla loro ricezione manoscritta, con particolare attenzione all'iconografia. L'articolo è corredato di 17 illustrazioni e di una tavola che elenca in ordine cronologico i manoscritti contenenti preghiere, con i dati significativi che li riguardano.

15 Le riflessioni conclusive che riepilogano le linee di ricerca e i risultati emersi dalle indagini presentate nei vari contributi sono affidate a Ardis BUTTERFIELD (Epilogue, pp. 269-277). Completano il volume l'Index des manuscrits (pp. 279-286) e l'Index nominum et des ceuvres médiévales (pp. 287-304). 